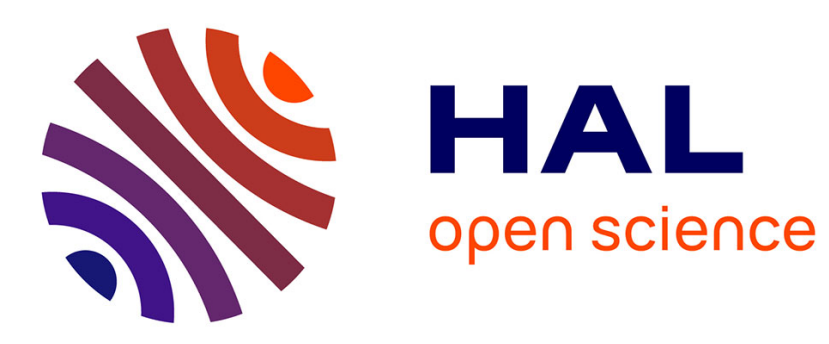

\title{
Kerker Effect with Hybridized Radiofrequency Resonators
}

\author{
Marc Dubois, Redha Abdeddaim, Stefan Enoch
}

\section{To cite this version:}

Marc Dubois, Redha Abdeddaim, Stefan Enoch. Kerker Effect with Hybridized Radiofrequency Resonators. 2018 IEEE Radio and Antenna Days of the Indian Ocean (RADIO), Oct 2018, Grand Port, Mauritius. pp.1-2, 10.23919/RADIO.2018.8572359 . hal-02471914

\section{HAL Id: hal-02471914 https://hal.science/hal-02471914}

Submitted on 12 Mar 2020

HAL is a multi-disciplinary open access archive for the deposit and dissemination of scientific research documents, whether they are published or not. The documents may come from teaching and research institutions in France or abroad, or from public or private research centers.
L'archive ouverte pluridisciplinaire HAL, est destinée au dépôt et à la diffusion de documents scientifiques de niveau recherche, publiés ou non, émanant des établissements d'enseignement et de recherche français ou étrangers, des laboratoires publics ou privés. 


\title{
Kerker effect with hybridized radiofrequency resonators
}

\author{
Marc Dubois, Redha Abdeddaim and Stefan Enoch \\ Aix Marseille Univ, CNRS, Centrale Marseille, Institut Fresnel \\ Marseille, France \\ marc.dubois@fresnel.fr
}

\begin{abstract}
A set of hybridized resonators is used to achieve efficient and tunable far field scattering control in the radiofrequency range. We demonstrate theoretically a full overlap between the electric dipolar and magnetic dipolar resonances of the meta-atom. This interaction is precisely tuned to reach the so-called Kerker scattering conditions when illuminated by a plane wave. Future work will tackle the illumination from a near field distributed source as the final goal of this study is to insert this element to control the radiofrequency electromagnetic field within a MRI birdcage coil.
\end{abstract}

\section{INTRODUCTION}

A crucial aspect for the control of radiation lies in the ability to tailor simultaneously the electric and magnetic responses of a single scatterer. This was first theoretically envisioned by Kerker et al. [1]: for a particle with specific values of permittivity and permeability, the fields scattered by the induced electric and magnetic dipoles can interfere thus leading to strong scattering anisotropy. This scattering anisotropy was recently measured in the microwave regime [2] and is referred as first and second Kerker conditions corresponding to a zero-backward scattering and a near-zero forward scattering depending on the excitation frequency. The Kerker conditions can also be extended to the case of a near field excitation when considering an electric dipole emitter coupled with a scatterer with strong electric and magnetic responses [3]. In this work, we demonstrate that the far field Kerker scattering conditions can be achieved by a compact meta atom. A meta atom can be designed using several resonant elements to build up a more complex unit cell using coupling mechanism [4]. The meta atom considered is based on a set of four hybridized resonant metallic wires. It will be referred as hybridized meta atom (HMA). First, we use an analytic approach based on impedance matrices in order to characterize the hybridization mechanism and derive the Kerker conditions. This model is then used to describe the interaction between the HMA and a plane wave excitation.

\section{IMPEDANCE MATRIX APPROACH AND FARFIELD STUDY}

A structure composed of several coupled resonators will present a new set of hybrid resonances. This effect is particularly striking when strong near field coupling occurs as the distance between resonators decreases with respect to the wavelength considered. We apply this strategy in order to design our HMA structure with strong electric and magnetic effective induced dipoles. It is composed of four z-oriented thin metallic wires located at the corners of a rectangle of sides $d_{1}=1.5 \mathrm{~cm}$ and $\mathrm{d}_{2}=2 \mathrm{~cm}$. A single wire mainly interacts with the electric field along its axis and presents an electric resonance when its length approaches a multiple of half a wavelength. However, when several wires are used with subwavelength separation, the coupling or hybridization between the wires cannot be neglected when describing the response of the whole structure.
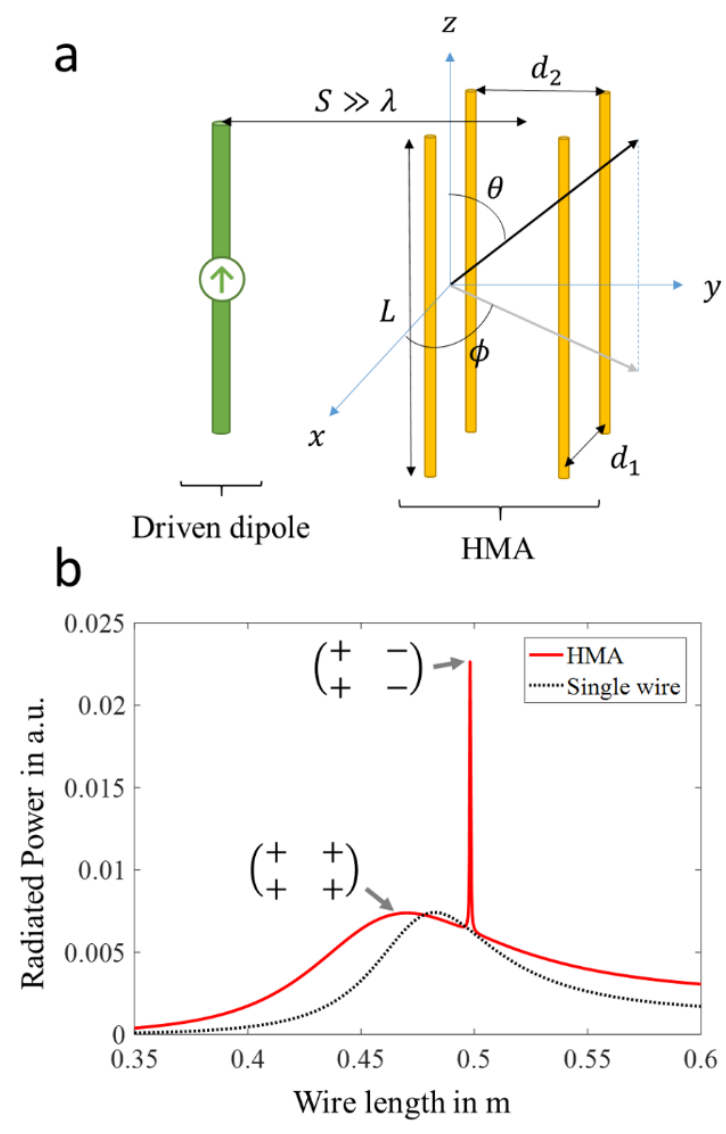

Fig. 1. (a) Sketch of the HMA configuration. A fifth wire is used as a source located at a distance $S$ large compared to the wavelength at $297.2 \mathrm{MHz}$. Such a configuration allows us to study the response of the HMA with a plane wave excitation. All wires have a diameter of $2 \mathrm{~mm}$. (b) Radiated power from the HMA (solid red) as a function of the wire length is compared with the power radiated by a single wire (dash black) of the same varying length. The inset matrices show the phase of the current within the four wires of the HMA.

The impedance matrix approach can be used to predict the interaction between array elements [5]. Within the assumption of sinusoidal current distribution, we are able to analytically derive the impedance matrix of the HMA and its coupling to a far field 
source. Once the current is known on every element, we are able to compute the radiation intensity in every directions as well as the total radiated power. Fig. 1 presents the geometry of the problem and the radiated power in function of the HMA length while the frequency is fixed to $300 \mathrm{MHz}$. The HMA response is characterized by a first broad peak corresponding to a current flowing in phase in all elements. This resonance resembles a normal dipolar electric mode. A second sharp peak appears at $\mathrm{L}=0.5 \mathrm{~m}$. At this length the current distribution is asymmetric and yields a magnetic dipole contribution. As this mode stores magnetic field within the small volume between the wires, it presents a large quality factor.
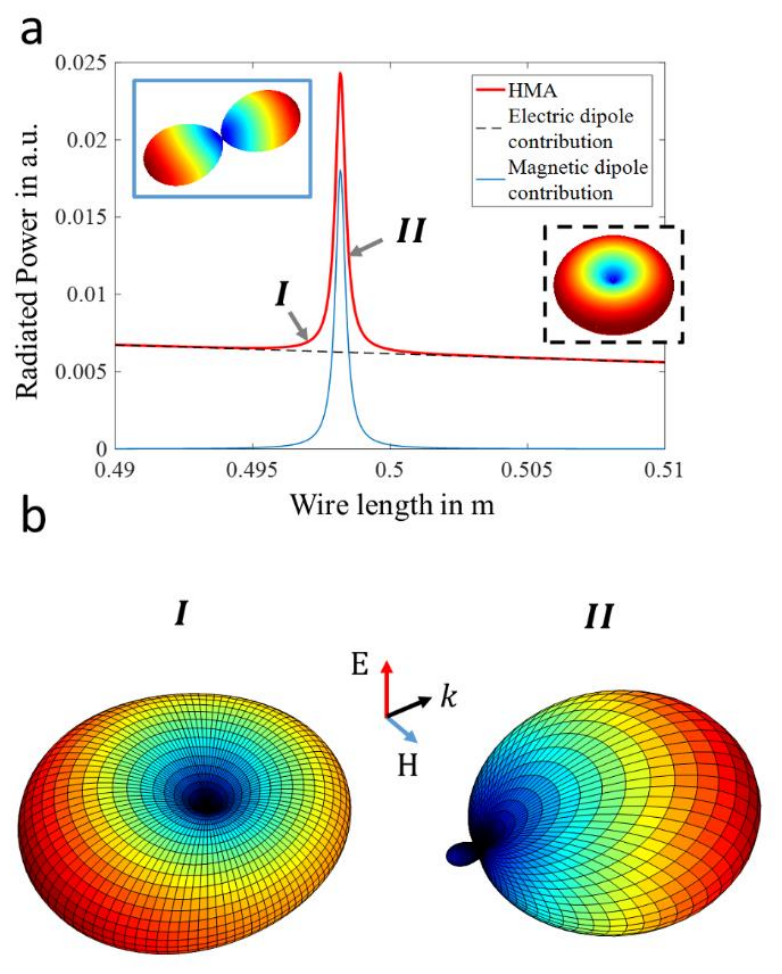

Fig. 2. (a) Radiated power from the HMA on a narrow window (solid red). Dash black line and solid thin blue line show the isolated contributions of the two resonances excited in the HMA. The two insets show the radiation pattern of the magnetic dipolar resonance and the electric dipolar resonance. (b) Far field radiation pattern at the two Kerker conditions (HMA lengths denoted above). The first one presents a reduction of the forward scattered field while a cancellation of the backward scattered field is observed at the second position.

\section{ISOLATION OF EFFECTIVE ELECTRIC AND MAGNETIC CONTRIBUTIONS}

With our method, it is possible to decompose the HMA response onto its two main contributions labelled electric and magnetic with respect to the current symmetry in the HMA structure. Fig. 2 shows the result of this decomposition on a narrow window of wire lengths. The insets in Fig. 2a show the two radiation patterns obtained when the contributions are isolated. The electric dipolar resonance resembles a donut as the current distribution is invariant by rotation around the $z$ axis. This is not the case for the magnetic dipolar resonance which shows a variation on both angles. Strong scattering anisotropy is obtained when these two behaviors are combined for wire lengths of $49.6 \mathrm{~cm}$ and $49.8 \mathrm{~cm}$ (Fig. $2 \mathrm{~d}$ ), due to interference between the two overlapping resonances. These specific lengths correspond to the Kerker conditions: a reduction of the forward scattering $(49.6 \mathrm{~cm})$ and a cancellation of the backward scattering $(49.8 \mathrm{~cm})$. They are obtained for precise interaction of electric and magnetic responses within the HMA. A detailed derivation of the conditions based on the existence of electric and magnetic dipolar modes in dielectric particles can be found in [6]. This is the first demonstration of scattering anisotropy or Kerker conditions from a single compact HMA

\section{CONCLUSION}

While these theoretical predictions will be validated with experimental measurements. Future work will focus on the excitation of the passive HMA structure from a distributed source in the near field. This source will model the field pattern of a standard MRI birdcage coil. We propose to use this type of structure in order to control the radiofrequency field within the birdcage coil which is a strong bottleneck for the use of MRI on human subject at ultra-high magnetic field (7Teslas and above).

\section{ACKNOWLEDGEMENT}

This work has received funding from the European Union's Horizon 2020 Research and Innovation programme under Grant Agreement No 736937.

\section{REFERENCES}

[1] M. Kerker, D.S. Wang and C.L. Gilles, "Electromagnetic scattering by magnetic spheres", JOSA, vol. 73, pp. 765-767, 1983.

[2] J.M. Geffrin, B. García-Cámara, R. Gómez-Medina, P. Albella, L.S. FroufePérez, C. Eyraud, A. Litman, R. Vaillon, F. González, M. Nieto-Vesperinas, J.J. Sáenz and F. Moreno, "Magnetic and electric coherence in forwardand back-scattered electromagnetic waves by a single dielectric subwavelength sphere", Nature Communications, vol. 3, pp. 1171, 2012.

[3] B. Rolly, J.M. Geffrin, R. Abdeddaim, B. Stout and N. Bonod, "Controllable emission of a dipolar source coupled with a magneto-dielectric resonant subwavelength scatterer", Scientific Reports, vol. 3, pp. 3063, 2012.

[4] N. Meinzer, W.L. Barnes, and I.R. Hooper, "Plasmonic meta-atoms and metasurfaces", Nature Photonics, vol. 8, pp. 889-898, 2014.

[5] S.J. Orfanidis, Electromagnetic waves and antennas, Rutgers University New Brunswick, NJ, 2002.

[6] M. Nieto-Vesperinas, R. Gomez-Medina, and J. J. Saenz, "Anglesuppressed scattering and optical forces on submicrometer dielectric particles", JOSA A, vol. 28, pp. 54-60, 2011. 